\title{
SOFT X-RADIATION FROM SINGLE ACTIVE REGIONS
}

\author{
D. H. BRABBA N*, E. B. DORLING, W. M. GLENCROSS, and J. R. H. HERRING \\ Mullard Space Science Laboratory, Dept. of Physics and Astronomy, \\ University College London, WCIE 6BT, England
}

Summary. The MSSL/Leicester University package on OSO 5 contained proportional counters having fields of view small compared with the area of the solar disk (Herring et al., 1971). Results discussed here were obtained with a detector sensitive in the band $0.3-0.9 \mathrm{~nm}$. This had an entrance window collimated to examine a strip of angular width 2' lying across the Sun.

One mode of operation allowed the field of view to remain stationary across a solar diameter. Observations of single active regions within this field showed that flare-like brightenings could be recognised most of the time, although changes in intensity of radiation at the detector might be as low as $10^{-5} \mathrm{erg} \mathrm{cm}^{-2} \mathrm{~s}^{-1}$ (Glencross et al., 1974). It appears that the bulk of the $\mathrm{X}$-ray emission from any active region might be produced by small flares, even when the area is regarded as 'relatively inactive'. In that case the mechanism responsible for flaring might be the one which is mainly responsible for heating the corona in active regions.

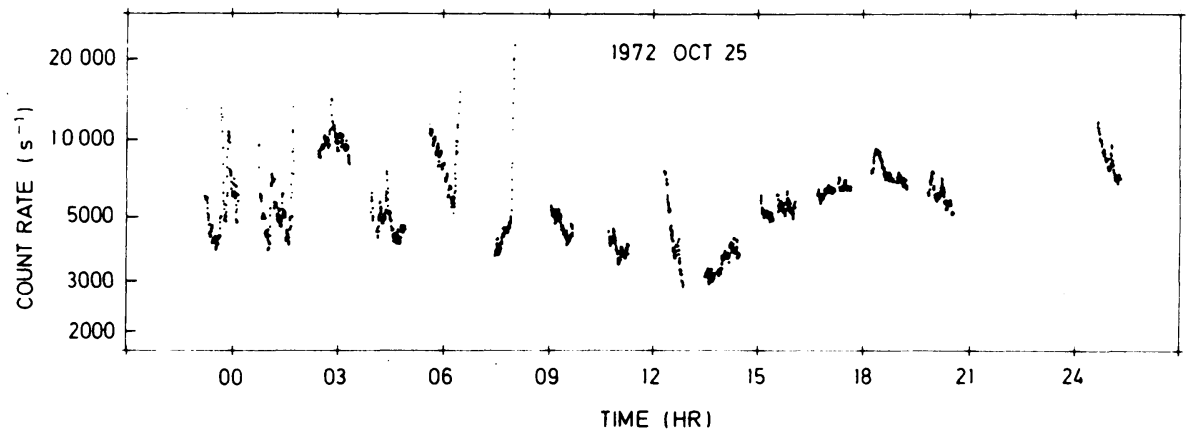

Fig. 1. Soft X-ray emission from McMath region 12094: After a period of considerable flaring, the flux of X-rays in the waveband $0.3-0.9 \mathrm{~nm}$ began to increase gradually at $1300 \mathrm{UT}$. This suggests there might be a common mechanism for heating coronal plasma during 'quiet' periods as well as during flaring.

Work is now in progress on an examination of long-term changes in flux from single active regions. Figure 1 shows data obtained from McMath region 12094 during one day. Although the region showed frequent flare brightenings until 1300 UT (optical data and the Solrad satellite show that events peaked at 1008 UT and $1136 \mathrm{UT}$, although OSO 5 was not operating during these periods), this was followed directly

* Now at Department of Space Research, University of Birmingham, U.K. 
by an enhancement of the baseline flux on which no significant short-lived increases developed. It will be argued in a paper being prepared that such changes in the character of the emission suggest once again that there might be a common mechanism for heating coronal plasma during 'quiet' periods as well as during flaring.

\section{References}

Glencross, W. M., Dorling, E. B., and Herring, J. R. H.: 1974, Solar Phys. 38, 183.

Herring, J. R. H., Glencross, W. M., Parkinson, J. H., and Pounds, K. A.: 1971, Proc. Roy. Soc. Lond. A 321, 493. 